\title{
Fe II FLUORESCENCE IN SYMBIOTIC STARS
}

\author{
M. Eriksson, ${ }^{1,2}$ H. Veenhuizen, ${ }^{2}$ G. M. Wahlgren, ${ }^{1}$ and S. Johansson ${ }^{1}$
}

\section{RESUMEN}

Se ha investigado la fluorescencia por PAR del ion Fe II en ocho estrellas simbióticas con muy diversos valores de la temperatura de la componente caliente y del período orbital. Los datos empleados son espectros de archivo del IUE. Todas las líneas de bombeo estudiadas se encuentran en la región (1200-2000 $\AA$ ) del IUE, excepto He II $\lambda 1084.942$ y O VI $\lambda 1032.041$. Las líneas de fluorescencia del Fe II están principalmente en la región Fe II, pero algunas caen en la región de las líneas de bombeo. Nuestro objetivo es entender las condiciones óptimas para la formación de líneas de fluorescencia por PAR del Fe II. Tres de los sistemas estudiados, RR Tel, AG Peg and V1016 Cyg, presentan 10-30 canales activos del Fe II. Dos condiciones relacionan a estos sistemas. La componente caliente es una enana blanca de temperatura extrema $\left(80 \cdot 10^{3}-150 \cdot 10^{3} \mathrm{~K}\right)$, y los tres sistemas son novas simbióticas, que han tenido erupciones en los últimos 150 años. Los sistemas AG Dra, RW Hya and R Aqr tienen sólo 2-3 canales activos del Fe II. Los dos sistemas restantes, CI Cyg and T CrB, no mostraron líneas de fluorescencia del Fe II. Estos sistemas tienen en común que la intensidad de emisión de los elementos altamente ionizados es menor que en la mayoría de los sistemas simbióticos, y que la componente caliente puede ser una estrella de secuencia principal con acreción, en vez de una enana blanca.

\section{ABSTRACT}

Fe II fluorescence by PAR has been investigated in eight symbiotic stars having a wide range in temperature of the hot component and orbital period. The data used are spectra obtained from the IUE archive. All pumping lines investigated in this work are in the short wavelength region of IUE (1200-2000 $\AA$ ), except for He II $\lambda 1084.942$ and O VI $\lambda 1032.041$. The resulting Fe II fluorescence lines are mainly in the long wavelength region (2000-3300 $\AA$ ), but a few fall in the same region as the pumping lines. The aim is to understand the optimal conditions for formation of Fe II fluorescence lines caused by PAR. Three of the selected systems, RR Tel, AG Peg and V1016 Cyg, have 10-30 active Fe II channels. Two conditions connect those systems to each other: The hot component is a white dwarf of extreme temperature $\left(80 \cdot 10^{3}-150 \cdot 10^{3} \mathrm{~K}\right)$ and all three systems are so called symbiotic novae and have had outbursts during the last 150 years. Three systems, AG Dra, RW Hya and R Aqr, have only 2-3 active Fe II channels. In the two remaining systems, CI Cyg and T $\mathrm{CrB}, \mathrm{Fe}$ II fluorescence lines were totally absent. These two systems have two features in common: The emission strength of highly ionized elements is less than in most symbiotic systems, and the hot component is suspected to be an accreting main sequence star rather than a white dwarf.

Key Words: ATOMIC PROCESSES - BINARIES: SYMBIOTIC

\section{INTRODUCTION}

Symbiotic stars are a subgroup of interacting binaries, and consist of a red giant, a hot UV source and a surrounding nebula. The orbital period of such a system is typically a few years, and the hot source (usually a white dwarf) is accreting material, either from the red giant wind, or by mass transfer caused by Roche lobe overflow from the red giant in some systems. This leads to accretion discs and sometimes even jets (Kafatos et al. 1987). Between the two stars there is a mechanically heated region caused by

\footnotetext{
${ }^{1}$ Atomic Astrophysics, Lund Observatory, Lund University, Box 43, SE-221 00, Lund, Sweden. (mattias@astro.lu.se).

${ }^{2}$ University College of Kalmar, SE-391 82, Kalmar, Sweden. (email:hans.veenhuizen@hik.se).
}

the collision of the winds from the two components (Mürset, Wolff \& Jordan 1997). A few of the symbiotic stars have had nova-like outbursts, followed by a slow decline lasting decades, and are referred to as symbiotic novae. This complexity leads to regions with very different temperatures and ionization stages, which increases the probability of PAR (Photoexcitation by Accidental Resonance) processes being observed. The most famous PAR process is the Bowen mechanism (Bowen 1935). In the symbiotic stars RR Tel and V1016 Cyg ten Fe II lines from high energy levels were explained as being pumped by PAR through the channel $a^{4} \mathrm{~F}_{9 / 2}-\mathrm{y}^{4} \mathrm{H}_{11 / 2}$ at $1548.204 \AA$ by the CIV $\lambda 1548.187$ line (Johansson 1983). Eriksson, Johansson \& Wahlgren (2001) 


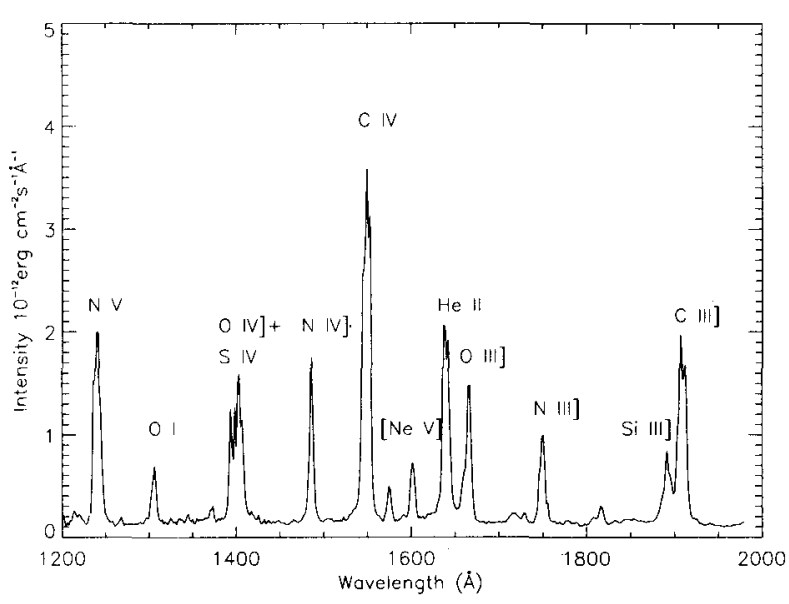

Fig. 1. Low resolution IUE spectrum number swp02427 of V1016 Cyg, showing the strong emission lines of highly ionized elements typical for symbiotic systems.

demonstrated that this PAR channel was active in six additional symbiotic stars: AG Peg, EG And, SY Mus, RX Pup, RW Hya and Z And. The Fe II UV emission lines of RR Tel and AG Peg have been analyzed (Hartman \& Johansson 2001; Eriksson, Johansson \& Wahlgren 2003), revealing 27 pumped Fe II channels in RR Tel and 30 in AG Peg with 120 and $180 \mathrm{Fe}$ II fluorescence lines, respectively. PAR is hence not a rare phenomenon but a dominant characteristic for the UV spectra of these two stars.

In this work the UV emission line spectra of RR Tel and AG Peg, along with six additional symbiotic systems, CI Cyg, V1016 Cyg, RW Hya, AG Dra, R Aqr and T CrB, were analyzed. The aim is to learn if Fe II pumping by PAR is common in symbiotic systems or if the numerous active Fe II channels make a special case for only a few systems. By comparing the results of the different symbiotic stars we also hope to see what conditions are ideal for PAR in symbiotic systems.

\section{FE II LEVELS PUMPED BY HIGHLY IONIZED ELEMENTS.}

At short wavelengths $(1000-2000 \AA)$ most of the strong lines originate from more ionized elements (Fig 1). These lines are most probably formed either in the hot wind from the white dwarf or in regions of the system ionized by the UV radiation from the white dwarf. If such a region irradiates a Fe II region the $\mathrm{Fe}^{+}$ions can start to fluoresce by PAR mechanisms.

Fe II levels pumped by highly ionized elements were observed in six of the eight selected systems and 23 different pumping channels were observed.
TABLE 1

FE II LEVELS PUMPED BY LINES FROM HIGHLY IONIZED ELEMENTS

\begin{tabular}{lll}
\hline Pumping line & Pumped level & $\lambda_{c h}{ }^{\mathrm{a}}(\AA)$ \\
\hline Si III] $\lambda 1892.030$ & $\mathrm{z}^{4} \mathrm{G}_{9 / 2}$ & 1892.078 \\
& $\mathrm{x}^{4} \mathrm{~F}_{5 / 2}$ & 1892.179 \\
N IV $\lambda 1718.550$ & $\mathrm{z}^{4} \mathrm{G}_{5 / 2}$ & 1718.101 \\
O III] $\lambda 1666.150$ & $\mathrm{y}^{4} \mathrm{P}_{5 / 2}$ & 1666.179 \\
O III] $\lambda 1660.809$ & $\mathrm{z}^{2} \mathrm{G}_{9 / 2}$ & 1660.839 \\
He II $\lambda 1640.474$ & $\mathrm{y}^{4} \mathrm{G}_{5 / 2}$ & 1640.152 \\
[Ne V] $\lambda 1575.129$ & $\mathrm{x}^{4} \mathrm{G}_{7 / 2}$ & 1574.772 \\
C IV $\lambda 1548.187$ & $\mathrm{y}^{4} \mathrm{H}_{11 / 2}$ & 1548.204 \\
& $\mathrm{y}^{2} \mathrm{D}_{5 / 2}$ & 1548.679 \\
& $\mathrm{w}^{2} \mathrm{D}_{3 / 2}$ & 1548.411 \\
& $\mathrm{y}^{6} \mathrm{~F}_{7 / 2}$ & 1548.028 \\
N IV] $\lambda 1486.496$ & $\mathrm{u}^{4} \mathrm{~F}_{3 / 2}$ & 1486.479 \\
O IV] $\lambda 1401.157$ & $\left({ }^{3} \mathrm{D}\right) 4 \mathrm{p}{ }^{4} \mathrm{P}_{3 / 2}$ & 1401.044 \\
Si IV $\lambda 1393.755$ & $\mathrm{x}^{2} \mathrm{H}_{9 / 2}$ & 1393.814 \\
N V $\lambda 1242.804$ & $\mathrm{v}^{2} \mathrm{G}_{7 / 2}$ & 1242.741 \\
N V $\lambda 1238.821$ & $\left({ }^{4} \mathrm{P}\right) 4 \mathrm{~s} 4 \mathrm{p}{ }^{4} \mathrm{P}_{5 / 2}$ & 1238.584 \\
O V] $\lambda 1218.344$ & $\left(\mathrm{~b}^{3} \mathrm{~F}\right) 4 \mathrm{p}{ }^{4} \mathrm{G}_{9 / 2}$ & 1218.213 \\
He II $\lambda 1084.942$ & $\mathrm{x}^{4} \mathrm{H}_{7 / 2}$ & 1085.903 \\
& $\mathrm{x}^{4} \mathrm{H}_{9 / 2}$ & 1085.579 \\
& $\mathrm{x}^{4} \mathrm{H}_{11 / 2}$ & 1084.992 \\
& $\mathrm{u}^{2} \mathrm{G}_{9 / 2}$ & 1084.932 \\
O VI $\lambda 1031.912$ & $\mathrm{u}^{2} \mathrm{G}_{7 / 2}$ & 1084.388 \\
& $\left(\mathrm{a}^{3} \mathrm{~F}\right) 5 \mathrm{p}{ }^{4} \mathrm{D}_{5 / 2}$ & 1032.041 \\
\hline
\end{tabular}

${ }^{a}$ Wavelength of the Fe II channel.

The pumped levels are listed with their corresponding pumping line in Table 1.

\section{FE II LEVELS PUMPED BY H I $\lambda 1215.671$}

The $\mathrm{H}$ Ly $\alpha$ line is strong and broad in cool giant stars where it has been observed to pump Fe II to high odd levels (Johansson \& Jordan 1984). In symbiotic systems the Lyman emission originates from the cool red giant atmosphere and not from the hot regions responsible for the Fe II fluorescence discussed in the previous section.

Among the eight selected symbiotic stars numerous H Ly $\alpha$ pumped Fe II levels were observed (Table 2). Atomic physics details are given in the study of RR Tel by Hartman \& Johansson (2000). Three systems, RR Tel, AG Peg and V1016 Cyg, have spectra showing lines originating from such levels. All three of these systems have had a nova outburst during the last 150 years. Since none of the other five 
TABLE 2

FE II LEVELS PUMPED BY H I $\lambda 1215.671$

\begin{tabular}{llll}
\hline Lower lev. & Pumped lev. & $\lambda_{c h}(\AA)$ & obs $^{\mathrm{a}}$ \\
\hline $\mathrm{a}^{4} \mathrm{D}_{7 / 2}$ & $\left({ }^{5} \mathrm{D}\right) 5 \mathrm{p}{ }^{6} \mathrm{~F}_{9 / 2}$ & 1217.848 & $\mathrm{R}, \mathrm{A}, \mathrm{V}$ \\
$\mathrm{b}^{4} \mathrm{P}_{3 / 2}$ & $\left({ }^{4} \mathrm{P}\right) 4 \mathrm{sp}{ }^{2} \mathrm{~S}_{1 / 2}$ & 1217.205 & $\mathrm{~A}$ \\
$\mathrm{a}^{4} \mathrm{D}_{3 / 2}$ & $\left(\mathrm{~b}^{3} \mathrm{P}\right) 4 \mathrm{p}{ }^{4} \mathrm{P}_{1 / 2}$ & 1217.152 & $\mathrm{R}$ \\
$\mathrm{a}^{4} \mathrm{D}_{1 / 2}$ & $\left({ }^{5} \mathrm{D}\right) 5 \mathrm{p}{ }^{4} \mathrm{D}_{3 / 2}$ & 1216.523 & $\mathrm{R}, \mathrm{A}$ \\
$\mathrm{a}^{4} \mathrm{D}_{3 / 2}$ & $\left({ }^{5} \mathrm{D}\right) 5 \mathrm{p}{ }^{4} \mathrm{P}_{5 / 2}$ & 1216.239 & $\mathrm{R}, \mathrm{A}, \mathrm{V}$ \\
$\mathrm{a}^{4} \mathrm{D}_{5 / 2}$ & $\left(\mathrm{~b}^{3} \mathrm{P}\right) 4 \mathrm{p}{ }^{4} \mathrm{~S}_{3 / 2}$ & 1215.983 & $\mathrm{R}, \mathrm{A}, \mathrm{V}$ \\
$\mathrm{a}^{4} \mathrm{D}_{5 / 2}$ & $\left({ }^{5} \mathrm{D}\right) 5 \mathrm{p}{ }^{4} \mathrm{D}_{5 / 2}$ & 1215.852 & $\mathrm{R}, \mathrm{A}$ \\
$\mathrm{a}^{4} \mathrm{G}_{11 / 2}$ & $\left({ }^{2} \mathrm{~F}\right) 4 \mathrm{sp}{ }^{4} \mathrm{G}_{11 / 2}$ & 1215.183 & $\mathrm{R}, \mathrm{A}$ \\
$\mathrm{b}^{4} \mathrm{~F}_{9 / 2}$ & $\left({ }^{2} \mathrm{I}\right) 4 \mathrm{sp}{ }^{4} \mathrm{H}_{9 / 2}$ & 1215.058 & $\mathrm{~A}$ \\
$\mathrm{a}^{4} \mathrm{D}_{1 / 2}$ & $\left({ }^{5} \mathrm{D}\right) 5 \mathrm{p}{ }^{4} \mathrm{~F}_{3 / 2}$ & 1214.150 & $\mathrm{R}$ \\
$\mathrm{a}^{4} \mathrm{D}_{3 / 2}$ & $\left({ }^{5} \mathrm{D}\right) 5 \mathrm{p}{ }^{4} \mathrm{~F}_{5 / 2}$ & 1213.738 & $\mathrm{R}$ \\
\hline
\end{tabular}

${ }^{\mathrm{a}}$ Stars showing fluorescence lines: $\mathrm{R}=\mathrm{RR}$ Tel, $\mathrm{A}=\mathrm{AG}$ Peg and V=V1016 Cyg.

symbiotic systems showed any sign of $\mathrm{H} \mathrm{Ly} \alpha$ pumped Fe II levels, it seems as if an outburst changes the properties of the circumstellar environment so that $\mathrm{H}$ Ly $\alpha$ can reach the Fe II region.

\section{STARS WITH NUMEROUS FE II FLUORESCENCE LINES}

Three systems showed substantial Fe II fluorescence (Fig 2): RR Tel (120 Fe II fluorescence lines), AG Peg (180 lines) and V1016 Cyg (58 lines). The temperature of the white dwarfs of V1016 Cyg and $\mathrm{RR}$ Tel is extremely high, $145000 \mathrm{~K}$ for V1016 Cyg (Mürset \& Nussbaumer 1994) and $142000 \mathrm{~K}$ for RR Tel (Jordan, Mürset \& Warner 1993). The temperature of the white dwarf in AG Peg is $~ 86000 \mathrm{~K}$ (Altamore \& Cassatella 1997), which is more common for symbiotic stars. Orbital periods for the systems are very different: $\sim 2$ years for $A G P$ Peg and $\sim 9$ years for V1016 Cyg (Meier et al. 1994). However, these three systems all belong to the subclass of symbiotic novae, which is a class of symbiotic stars that have undergone slow nova outbursts. The outburst started in 1850 for AG Peg, in 1944 for RR Tel and in 1964 for V1016 Cyg.

\section{STARS WITH FEW FE II FLUORESCENCE LINES}

The five systems not classified as symbiotic novae have much fewer Fe II fluorescence lines. Only three of them had $\mathrm{Fe}$ II fluorescence lines at all, RW Hya (12 lines), R Aqr (6 lines) and AG Dra (5 lines). In RW Hya and AG Dra the only line pumping Fe II is C IV $\lambda 1548.187$ while Si III] $\lambda 1892.030$ and O III]

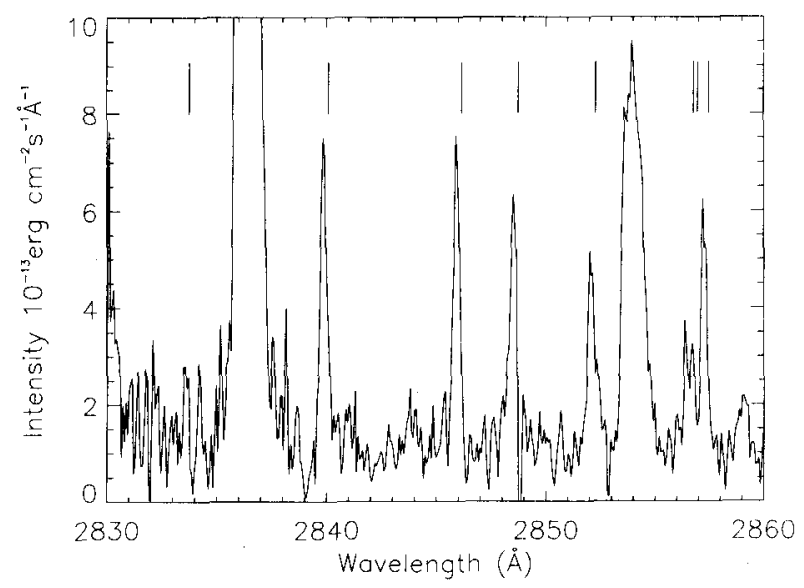

Fig. 2. High resolution IUE spectrum number LWP04961 of V1016 Cyg. All marked lines are Fe II fluorescence lines.

$\lambda 1666.150$ cause the Fe II fluorescence in R Aqr. The white dwarf temperature of RW Hya, $\sim 41000 \mathrm{~K}$ (Sion et al. 2002), and R Aqr, $\sim 61000 \mathrm{~K}$ (Meier \& Kafatos 1995), is low compared to the stars with many Fe II fluorescence lines. This indicates that the temperature of the white dwarf is important for PAR processes in symbiotic systems. However, the white dwarf in AG Dra has a temperature of $\sim 110000 \mathrm{~K}$ (González-Riestra et al. 1999) and does still have only a few Fe II lines.

\section{STARS WITH NO FE II FLUORESCENCE}

Two symbiotic systems, $\mathrm{CI}$ Cyg and $\mathrm{T} \mathrm{CrB}$, do not show any sign of Fe II fluorescence. The hotter component in $\mathrm{T} \mathrm{CrB}$ is suggested to be a main sequence star (Kenyon \& Garcia 1986), which accretes $\sim 10^{-6} \mathrm{M}_{\odot}$ per year from the cooler component leading to $\mathrm{T}_{\text {eff }} \sim 25000 \mathrm{~K}$ in the disc. This can explain the absence of Fe II fluorescence in $\mathrm{T} \mathrm{CrB}$ since the UV flux from the hot component would probably not create large amounts of $\mathrm{C}^{3+}, \mathrm{Si}^{2+}$, or $\mathrm{N}^{4+}$. $\mathrm{T}$ $\mathrm{CrB}$ is the most compact system among the eight selected symbiotic stars with a period of only 227.5 days. Even if the hotter component is a white dwarf, the UV flux from the hot component may not reach very far since it can be obscured by material expelled from the red giant. Also CI Cyg is suggested to have a hot component in the form of an accretion disc around a main sequence star (Iijima 1982). Thus, the UV flux of the hot component depends on the accretion rate from the red giant onto the disc. Kenyon et al. (1991) measured a mass transfer of a few times $10^{-5} \mathrm{M}_{\odot}$ per year onto the disc. Hence, the absence of $\mathrm{Fe}$ II fluorescence lines in the spectrum of CI Cyg can also depend on deficient UV flux in the system. 


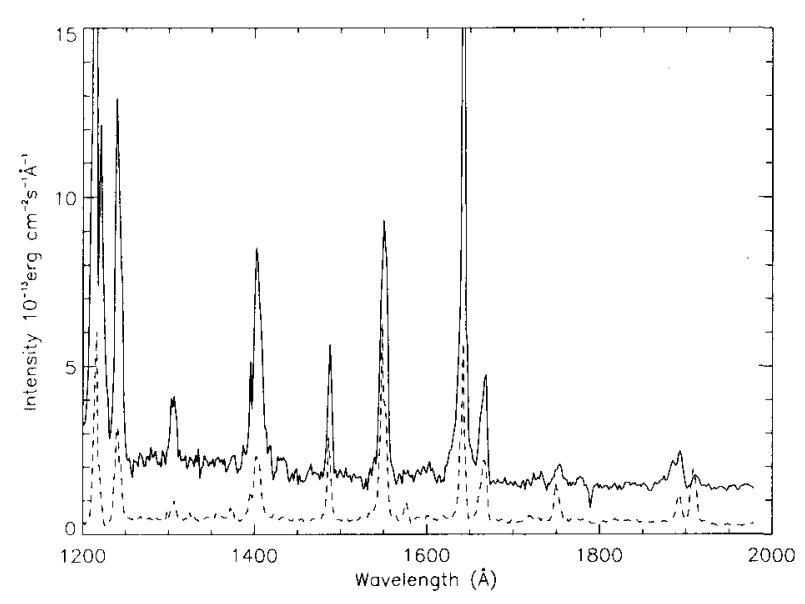

Fig. 3. Comparison between emission strength of highly ionized elements in AG Dra and CI Cyg. The solid line corresponds to the low resolution spectrum swp09084 of AG Dra and the dotted line to the low resolution spectrum swp36320 of CI Cyg. Notice also the difference in the UV continuum.

Both CI Cyg and T CrB have much weaker emission lines between $1200-2000 \AA$ from the highly ionized elements (Fig 3). This supports the idea of less ionizing UV flux leading to fewer regions of elements that can pump Fe II.

\section{SUMMARY}

The symbiotic stars in our selection can clearly be placed in three different groups with respect to Fe II fluorescence (Table 3):

- Symbiotic novae with white dwarfs of extremely high temperature.

- Symbiotic systems with white dwarfs of moderate temperature (AG Dra causes a problem because of the high temperature of the white dwarf).

- Symbiotic systems with an accreting main sequence star instead of a white dwarf.

Two conclusions can probably be drawn. Fe II fluorescence increases after a nova outburst and a white dwarf is needed in the system to obtain UV radiation enough to create regions of elements that can pump Fe II. However, to confirm the statements a larger number of stars have to be included in the analysis. It would also be of value to investigate whether the pumping of other elements such as Co II and O III, known to be pumped by PAR in symbiotic stars, follow the same trends as Fe II.

\section{REFERENCES}

Altamore, A., \& Cassatella A. 1997, A\&A, 317, 712
TABLE 3

THE NUMBER OF FEII CHANNELS AND

LINES RELATIVE TO THE WHITE DWARF TEMPERATURES.

\begin{tabular}{lrrrc}
\hline System & ch. $^{\text {a }}$ & lines $^{\mathrm{a}}$ & temp. $^{\mathrm{b}}$ & note $^{\mathrm{c}}$ \\
\hline AG Peg & 30 & 180 & 86 & syn \\
RR Tel & 27 & 120 & 145 & syn \\
V1016 Cyg & 10 & 58 & 142 & syn \\
\hline RW Hya & 3 & 12 & 41 & \\
R Aqr & 2 & 6 & 61 & \\
AG Dra & 2 & 5 & 110 & \\
\hline CI Cyg & 0 & 0 & & dis \\
T CrB & 0 & 0 & & dis \\
\hline
\end{tabular}

${ }^{a}$ Number of pumped Fe II channels and lines.

${ }^{b}$ Temperatures of the white dwarfs given in $10^{3} \mathrm{~K}$

${ }^{c}$ syn $=$ symbiotic novae, dis $=$ disc around main sequence star and not a white dwarf as hot component.

Bowen, I. S. 1935, ApJ, 81, 1

Eriksson, M., Johansson, S., \& Wahlgren, G. M. 2003, in ASP Conf. Ser. 303, Symbiotic stars probing stellar evolution, ed. R. L. M. Corradi, J. Mikolajewska, \& T. J. Mahoney (San Francisco: ASP), ????

Eriksson, M., Johansson, S., \& Wahlgren, G. M. 2001, in ASP Conf. Ser., 242, $\eta$ Carinae and other mysterious stars: the hidden opportunities of emission spectroscopy, ed. T. R. Gull, S. Johansson, \& K. Davidsson (San Francisco: ASP), 325

González-Riestra, R., Viotti, R., Iijima, T., \& Greiner, J. 1999, A\&A, 347, 478

Hartman, H., \& Johansson, S. 2000, A\&A, 359, 627

Iijima, T. 1982, A\&A, 116, 210

Johansson, S. 1983, MNRAS 205, 71

Johansson, S., \& Jordan, C. 1984, MNRAS, 210, 239

Jordan, S., Mürset, U., \& Werner, K. 1994, A\&A, 283, 475

Kafatos, M., Cassatella, A., Michalitsianos, A. G., Piro, L., \& Viotti, R. 1987, in IAU Symposium, 122, Circumstellar matter, ed. I. Appenzeller \& C. Jordan (Dordrecht: Reidel), 491

Kenyon, S. J., Oliversen, N. A., Mikolajewska, J., Mikolajewski, M., Stencel, R. E., Garcia, M. R,. \& Anderson, C. M. 1991, AJ, 101, 637

Kenyon, S. J., \& Garcia, M. R. 1986, AJ, 91, 125

Meier, S. R., Kafatos, M., Fahey, R. P., \& Michalitsianos, A. G. 1994, ApJS, 94, 183

Meier, S. R., \& Kafatos, M. 1995, ApJ, 451, 359

Mürset, U., Wolff, B., \& Jordan, S. 1997, A\&A, 319, 201

Mürset, U., \& Nussbaumer, H. 1994, A\&A, 282, 586, 1994

Sion, E. M., Mikolajewska, J., Bambeck, D., \& Dumm, T. 2002 , AJ, 123,983 


\section{DISCUSSION}

Kaper - Did you correlate the properties of the Fe II fluorescence lines with those of Raman scattered emission lines, as observed in these systems by Vogel, Nussbaumer et al.?

Eriksson - No. Raman scattered lines are much broader because of short lifetimes in associated virtual states. I suppose that environments positive for PAR also could be in favor of Raman scattering. If I remember correctly, Raman scattering was observed in the same systems where we found many Fe II pumping channels.

Mattias Eriksson, Glenn M. Wahlgren and Sveneric Johansson: Atomic Astrophysics, Lund Observatory, Lund University, Box 43, SE-221 00 Lund, Sweden. (mattias@astro.lu.se).

Hans Veenhuizen: University College of Kalmar, SE-391 82 Kalmar, Sweden. (hans.veenhuizen@hik.se). 